\title{
Exogenous administration of heme oxygenase-1 by gene transfer provides protection against hyperoxia-induced lung injury
}

\author{
Leo E. Otterbein, ${ }^{1,2}$ Jay K. Kolls, ${ }^{3}$ Lin L. Mantell, ${ }^{4}$ Julia L. Cook, Jawed Alam, ${ }^{5}$ \\ and Augustine M.K. Choi ${ }^{1}$
}

\begin{abstract}
${ }^{1}$ Section of Pulmonary and Critical Care Medicine, Yale University School of Medicine, New Haven, Connecticut 06250, USA ${ }^{2}$ The Johns Hopkins Medical Institutions, Baltimore, Maryland 21205, USA

${ }^{3}$ Gene Therapy Program, and Departments of Medicine and Pediatrics, Louisiana State University, New Orleans, Louisiana 70112, USA

${ }^{4}$ The CardioPulmonary Research Institute, Department of Thoracic Cardiovascular Surgery, Winthrop University Hospital, and State University of New York at Stony Brook School of Medicine, Mineola, New York 11501, USA

${ }^{5}$ Department of Molecular Genetics, Alton Ochsner Medical Foundation, and Department of Biochemistry and Molecular Biology, Louisiana State University Medical Center, New Orleans, Louisiana 70121, USA
\end{abstract}

Address correspondence to: Augustine M.K Choi, Section of Pulmonary and Critical Care Medicine, Yale University School of Medicine, 333 Cedar Street, LCI 105, New Haven, Connecticut 06520, USA. Phone: (203) 785-3627; Fax: (203) 937-4784;

E-mail: augustine.choi@yale.edu

Received for publication September 25, 1998, and accepted in revised form February 25, 1999.

\begin{abstract}
Heme oxygenase-1 (HO-1) confers protection against a variety of oxidant-induced cell and tissue injury. In this study, we examined whether exogenous administration of HO-1 by gene transfer could also confer protection. We first demonstrated the feasibility of overexpressing $\mathrm{HO}-1$ in the lung by gene transfer. A fragment of the rat $\mathrm{HO}-1 \mathrm{cDNA}$ clone containing the entire coding region was cloned into plasmid pAC-CMVPLPA, and recombinant adenoviruses containing the rat $\mathrm{HO}-1 \mathrm{cDNA}$ fragment Ad5-HO-1 were generated by homologous recombination. Intratracheal administration of Ad5-HO-1 resulted in a timedependent increase in expression of HO-1 mRNA and protein in the rat lungs. Increased HO- 1 protein expression was detected diffusely in the bronchiolar epithelium of rats receiving Ad5-HO-1, as assessed by immunohistochemical studies. We then examined whether ectopic expression of $\mathrm{HO}-1$ could confer protection against hyperoxia-induced lung injury. Rats receiving Ad5-HO-1, but not AdV- $\beta$ Gal, a recombinant adenovirus expressing Escherichia coli $\beta$-galactosidase, before exposure to hyperoxia $\left(>99 \% \mathrm{O}_{2}\right)$ exhibited marked reduction in lung injury, as assessed by volume of pleural effusion and histological analyses (significant reduction of edema, hemorrhage, and inflammation). In addition, rats receiving Ad5-HO-1 also exhibited increased survivability against hyperoxic stress when compared with rats receiving AdV- $\beta$ Gal. Expression of the antioxidant enzymes manganese superoxide dismutase (Mn-SOD) and copper-zinc superoxide dismutase (CuZn-SOD) and of $\mathrm{L}$-ferritin and $H$-ferritin was not affected by Ad5HO-1 administration. Furthermore, rats treated with Ad5-HO-1 exhibited attenuation of hyperoxiainduced neutrophil inflammation and apoptosis. Taken together, these data suggest the feasibility of high-level HO-1 expression in the rat lung by gene delivery. To our knowledge, we have demonstrated for the first time that HO-1 can provide protection against hyperoxia-induced lung injury in vivo by modu-
\end{abstract} lation of neutrophil inflammation and lung apoptosis.

J. Clin. Invest. 103:1047-1054 (1999).

\section{Introduction}

Heme oxygenase $(\mathrm{HO})$ catalyzes the first and rate-limiting step in the degradation of heme to yield equimolar quantities of biliverdin IXa, carbon monoxide (CO), and iron $(1,2)$. Biliverdin is then converted to bilirubin by biliverdin reductase (1). Three isoforms of $\mathrm{HO}$ exist: $\mathrm{HO}-$ 1 is highly inducible, whereas $\mathrm{HO}-2$ and $\mathrm{HO}-3$ are constitutively expressed $(2,3)$. In addition to heme, a variety of nonheme compounds, including heavy metals, cytokines, hormones, endotoxins, and heat shock, are strong inducers of HO-1 expression $(1,4)$. This chemical diversity of HO-1 inducers has led to the speculation that $\mathrm{HO}-1$, in addition to its role in heme degradation, may also serve a vital function in maintaining cellular homeostasis. Support for this hypothesis has been strengthened by accumulating evidence that $\mathrm{HO}-1$ is highly induced by a variety of agents causing oxidative stress, including hydrogen peroxide, glutathione depletors, ultraviolet irradiation, and hyperoxia (5-7).

Recent observations have demonstrated that increased endogenous HO-1 provides cellular protection against heme-mediated oxidant injury (8-10). In a rat model of rhabdomyolysis, Nath et al. (8) have observed that the prior induction of HO-1 with preinfusion of hemoglobin prevents kidney failure and reduces mortality. Otterbein et al. (9) have reported similar protective effects of HO-1 induction in a rat model of endotoxic shock and lung injury. Abraham et al. (10) have demonstrated that overexpression of HO-1 in cultured endothelial cells provides cellular protection against heme toxicity.

Recent studies from our laboratory have also demonstrated that induction of endogenous expression of 

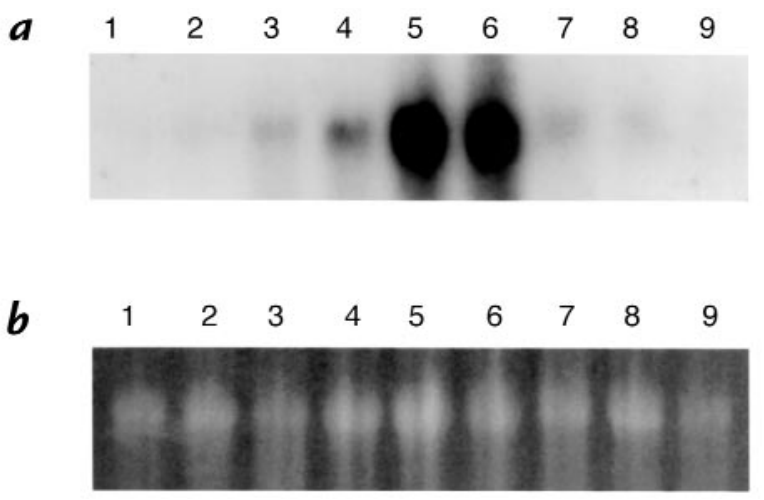

Figure 1

Kinetics of HO-1 mRNA expression in rat lungs after Ad5-HO-1 administration. Total RNA was extracted from lung tissue after Ad5-HO-1 administration and analyzed for HO-1 mRNA expression by Northern blot analysis at the indicated times. (a) Lane 1: vehicle control $72 \mathrm{~h}$; lane 2: vehicle control $72 \mathrm{~h}$; lane 3: $6 \mathrm{~h}$; lane 4: $24 \mathrm{~h}$; lane 5: $48 \mathrm{~h}$; lane 6: $72 \mathrm{~h}$; lane 7: 1 week; lane 8: 2 weeks; lane 9: 2 weeks. (b) Ethidium bromide staining of the same RNA is shown for loading control. HO-1, heme oxygenase-1.

HO- 1 confers protection against nonheme-mediated oxidative stress, including hyperoxia, in vitro (11). For example, overexpression of $\mathrm{HO}-1$ in lung epithelial cells protected cells against hyperoxia-induced cell death. In view of these data, we sought to examine whether exogenous administration of $\mathrm{HO}-1$ could directly provide protection against hyperoxic lung injury in vivo. In this study, we employ a recombinant adenovirus containing the rat HO-1 cDNA (Ad5-HO-1) to successfully overexpress $\mathrm{HO}-1$ in rat lung after intratracheal administration. We demonstrate that exogenous delivery of HO-1 by gene transfer can provide protection against hyperoxia-induced lung injury in vivo. We also provide data highlighting possible mechanisms responsible for this protection.

\section{Methods}

Generation of recombinant adenovirus containing HO-1 cDNA. A 1.0$\mathrm{kbp}$ XhoI-HindIII fragment from the rat HO-1 cDNA clone pRHO-1 (12), containing the entire coding region, was cloned into plasmid pAC-CMVPLPA $(13,14)$. Recombinant HO- 1 adenovirus Ad5-HO-1 was generated by homologous recombination in 911 cells (15) after cotransfection with the pAC-HO-1 recombinant plasmid and plasmid pJM17 (16). The recombinant Ad5-HO-1 clones were screened by Southern blot analysis (17). The recombinant adenovirus containing the Escherichia coli $\beta$-galactosidase gene, AdV- $\beta$ Gal, has been described (17). Isolation, propagation, and titering of recombinant adenoviruses were carried out as described previously $(13,17)$.

Adenovirus administration. Pathogen-free Sprague Dawley rats (225-250 g) were purchased from Harlan Sprague Dawley (Indianapolis, Indiana, USA) and allowed to acclimate upon arrival for 7 days before experimentation. Animals were fed rodent chow and water ad libitum. On the day of experimentation, each rat was anesthetized with $2 \% \mathrm{vol} / \mathrm{vol}$ isoflurane, and the trachea was exposed. Then $10^{9}$ plaque-forming units ( $\mathrm{pfu}$ ) of Ad5-HO- 1 or AdV- $\beta$ Gal was injected intratracheally in a volume of $200 \mu \mathrm{l}$. AdV- $\beta$ Gal served as vector control in all experiments. Additional animals receiving $10 \%$ glycerol (buffer) alone also served as vehicle controls for all experiments. The incision was closed with wound clips, and the rats were allowed to recover for $15 \mathrm{~min}$. At this time, one group was placed in hyperoxia while another was kept in normoxia.
Oxygen exposures. Animals were exposed to hyperoxia (>99\% $\mathrm{O}_{2}$ ) at a flow rate of $12 \mathrm{l} / \mathrm{min}$ in a $3.7-\mathrm{ft}^{2}$ Plexiglas exposure chamber and were supplied with rodent chow and water ad libitum throughout the exposures. One group of animals receiving either Ad5-HO-1, AdV- $\beta$ Gal, or $10 \%$ glycerol were placed in hyperoxia and monitored for survival. A second group was sacrificed at $56 \mathrm{~h}$ to determine pleural effusion volume, analysis of bronchoalveolar lavage (BAL) cell count, and histological analysis of lungs fixed with $10 \%$ buffered formalin for hematoxylin and eosin staining, as described previously (6).

Immunohistochemistry. Methods described previously were used for immunohistochemistry studies (18). Briefly, formalinfixed tissue sections were rehydrated with PBS and blocked with $2 \%$ nonfat dry milk before incubating with a 1:100 dilution of the primary antibody, anti-rat HO-1 (StressGen Biotechnologies Corp., Victoria, British Columbia, Canada), in $3 \%$ BSA overnight at $4^{\circ} \mathrm{C}$. Sections were washed three times with PBS (5 min each). The secondary antibody, a biotinylated goat anti-rabbit IgG, was incubated with a dilution of 1:500 in $3 \% \mathrm{BSA}$ at $37^{\circ} \mathrm{C}$ for $1 \mathrm{~h}$ and detected with peroxidase-conjugated avidin-biotin complex. Negative controls for nonspecific binding included normal rabbit serum without primary antibody or with secondary antibody alone. The slides were scanned with a Kodak Slide Scanner RFS-2035 (Eastman Kodak Co., Rochester, New York, USA) using the Photoshop software program (Adobe Systems, Inc., Mountain View, California, USA) and a color composite was printed using the Kodak Printer XL770 Digital Continuous Tone. The Photoshop software program was used to prepare the composite image.

RNA extraction and Northern blot analysis. Total RNA was isolated by the STAT-60 RNAzol method, with homogenization of the lung tissues in RNAzol lysis buffer followed by chloroform extraction (Tel-Test Inc., Friendswood, Texas, USA). Northern blot analyses were performed as described previously (18). Briefly, $10 \mathrm{mg}$ of total RNA was electrophoresed in a $1 \%$ agarose gel and transferred to Gene Screen Plus nylon membrane (Du Pont NEN Research Products, Boston, Massachusetts, USA) by capillary action. The nylon membranes were then prehybridized in hybridization buffer (1\% BSA, 7\% SDS, $0.5 \mathrm{M}$ phosphate buffer [pH 7.0], $1.0 \mathrm{mM}$ EDTA) at $65^{\circ} \mathrm{C}$ for $2 \mathrm{~h}$, followed by incubation with hybridization buffer containing ${ }^{32} \mathrm{P}$ labeled rat HO- $1 \mathrm{cDNA}$ at $65^{\circ} \mathrm{C}$ for $24 \mathrm{~h}$. Nylon membranes were then washed twice in wash buffer A $(0.5 \%$ BSA, $5 \%$ SDS, 40 $\mathrm{nM}$ phosphate buffer [pH 7.0], $1 \mathrm{mM}$ EDTA) for $15 \mathrm{~min}$ each at $65^{\circ} \mathrm{C}$, followed by washes in buffer $\mathrm{B}(1 \% \mathrm{SDS}, 40 \mathrm{mM}$ phosphate buffer [pH 7.0], $1.0 \mathrm{mM}$ EDTA) for $15 \mathrm{~min}$, three times each, at $65^{\circ} \mathrm{C}$. Ethidium bromide staining of the gel was used to confirm the integrity and equal loading of the RNA.

cDNA and oligonucleotide probes. A full-length rat HO-1 cDNA, generously provided by S. Shibahara (Sendai University, Tohoku, Japan) (12), was subcloned into pBluescript vector. A HindIII-EcoRI digestion was performed to cut the 0.9-kb HO-1

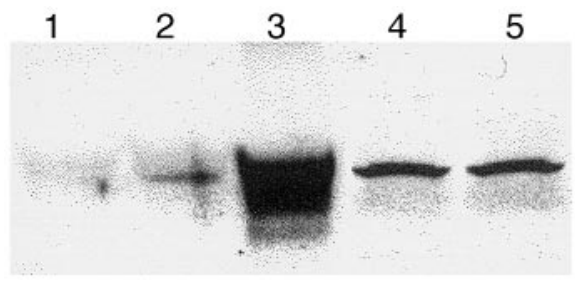

Figure 2

HO-1 protein expression in rat lungs after Ad5-HO-1 administration. Total protein was extracted from lung tissue after Ad5-HO-1 administration and analyzed for $\mathrm{HO}-1$ protein expression by Western blot analysis. Lane 1: vehicle control $72 \mathrm{~h}$; lane 2: AdV- $\beta$ Gal $72 \mathrm{~h}$; lane 3: Ad5-HO172 h; lane 4: Ad5-HO-1 1 week; lane 5: Ad5-HO-1 2 weeks. 


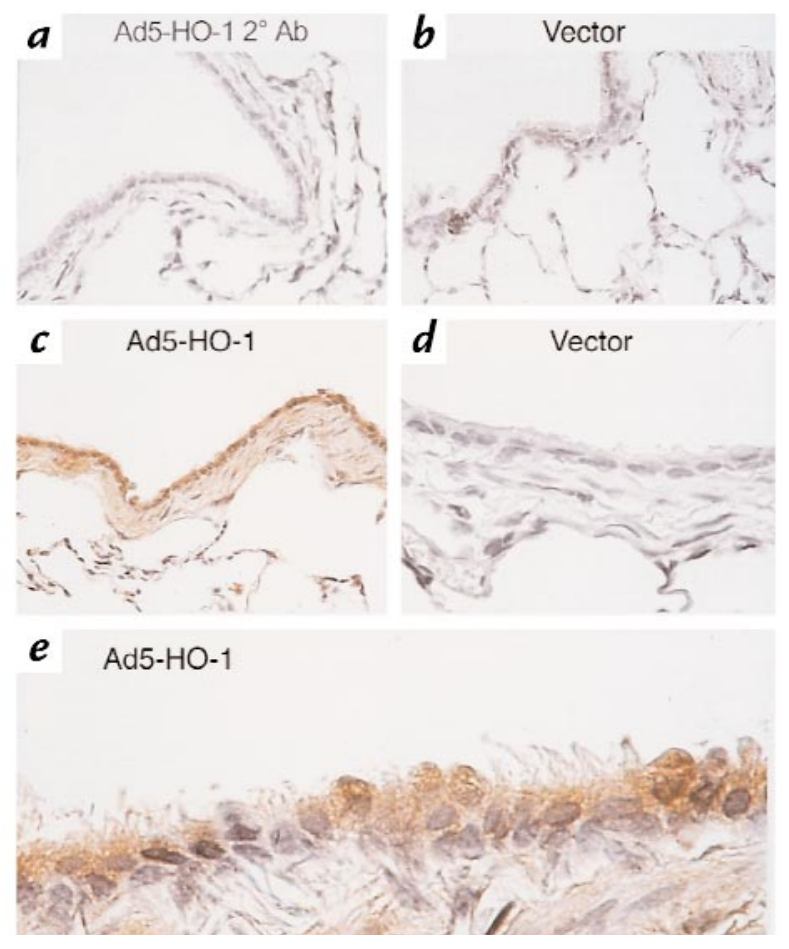

Figure 3

Immunohistochemical studies of the rat lung after Ad5-HO-1 treatment at $72 \mathrm{~h}$. Formalin-fixed sections were analyzed for $\mathrm{HO}-1$ expression as described in Methods. (a) Ad5-HO-1-treated rat lungs stained with secondary antibody alone. $\times 26$. (b) Vector control. $\times 26$. (c) Ad5-HO-1. $\times 26$. (d) Vector control. $\times 100 .($ e $)$ Ad5-HO-1. $\times 100$.

cDNA insert out of the pBluescript vector. Rat manganese superoxide dismutase (Mn-SOD) and copper-zinc superoxide dismutase (CuZn-SOD) cDNAs were generously provided by $\mathrm{H}$. Nick (University of Florida, Gainsville, Florida, USA). Oligonucleotide probes for rat L-ferritin 5'-GCCCAGAGAGAGGTAGGTGTAAGAGGCCCGCAGGTGCAAG-3') (20) and $H$-ferritin (5'-CCTGGTGGTAGTTCTGGCGCACTTGCGAGGGAGACGCGG-3') (19) were synthesized commercially by IDT Inc. (Coralville, Iowa, USA). To control for variation in either the amount of RNA in different samples or loading errors, blots were hybridized with an oligonucleotide probe corresponding to the 18s rRNA. A 24bp oligonucleotide (5'-ACG GTA TCT GAT CGT CTT CGA ACC-3') complementary to the 18 s RNA was synthesized using a DNA synthesizer (Applied Biosystems; Foster City, California, USA). The cDNAs were labeled with [ $\left.{ }^{32} \mathrm{P}\right] \mathrm{CTP}$, using the random primer kit from Boehringer Mannheim Biochemica (Mannheim, Germany), and oligonucleotides were labeled with [ $\left.{ }^{32} \mathrm{P}\right] \mathrm{ATP}$ at the $3^{\prime}$ end with terminal deoxynucleotidyl transferase (Bethesda Research Laboratories, Gaithersburg, Maryland, USA).

Western blot analysis. Frozen tissues were homogenized in lysis buffer containing NP-40 (10\%). Protein concentrations of the lysates were determined by Coomassie blue dye-binding assay (Bio-Rad Laboratories Inc., Hercules, California, USA). An equal volume of double-strength SDS/sample buffer (0.125 M Tris$\mathrm{HCl}$ [pH 7.4], 4\% SDS, and 20\% glycerol) was added, and the samples were boiled for $5 \mathrm{~min}$. Samples were subjected to electrophoresis in a $12 \%$ SDS-polyacrylamide gel (Novex, San Diego, California, USA) for $2 \mathrm{~h}$ at $20 \mathrm{~mA}$. The gel was transferred electrophoretically (Bio-Rad Laboratories Inc.) onto a polyvinylidene fluoride membrane (Immobilon-P; Millipore Corp., Bedford, Massachusetts, USA) and incubated for $2 \mathrm{~h}$ in Tris-buffered saline containing $1 \%$ polyoxyethylene sorbitan monolaurate (TTBS) and 5\% nonfat powdered milk. The mem- branes were then incubated for $2 \mathrm{~h}$ with rabbit polyclonal antibody against rat HO-1 (1:1,000 dilution). After washes in TTBS three times for $5 \mathrm{~min}$ each, the membranes were incubated with goat anti-rabbit IgG antibody (Amersham Life Sciences Inc., Arlington Heights, Illinois, USA) for $2 \mathrm{~h}$. The membranes were then washed three times in TTBS for 5 min each, followed by signal development using an enhanced chemiluminescence (ECL) detection kit from Amersham.

Lung tissue preparation. Lungs were fixed by perfusion of $10 \%$ formalin at $20 \mathrm{~cm} \mathrm{H}_{2} 0$ gauge pressure and embedded in paraffin. Lung sections at 4-5 $\mathrm{mM}$ were mounted onto slides pretreated with 3-aminopropylethoxysilane (Digene Diagnostics Inc., Silver Spring, Maryland, USA). Slides were baked for $30 \mathrm{~min}$ at $60^{\circ} \mathrm{C}$ and washed twice in fresh xylenes for $5 \mathrm{~min}$ each to remove the paraffin. The slides were then rehydrated though a series of graded alcohols and then washed in distilled water for 3 min each.

$B A L$ analysis. Animals were anesthetized with sodium pentobarbital after $56 \mathrm{~h}$ of hyperoxia exposure. BAL ( $35 \mathrm{ml} / \mathrm{kg}$ ) was performed four times with PBS ( $\mathrm{pH}$ 7.4). Cell pellets were pooled from the lavages and centrifuged at $1,200 \mathrm{~g}$ for $10 \mathrm{~min}$. The supernatant was discarded and cells were resuspended in PBS. Cell counts were performed using a Neubauer hemocytometer (VRW, Boston, Massachusetts, USA). For differential analysis, samples were cytocentrifuged and stained with DiffQuik (Fischer Scientific, Pittsburgh, Pennsylvania, USA).

Apoptosis by TUNEL assay and photomicrography. The terminal deoxynucleotide transferase-mediated dUTP nick end-labeling (TUNEL) method was used for apoptosis assay of lung tissue sections as described previously (210). TUNEL reagents including rhodamine-conjugated antidigoxigenin Fab fragment were obtained from Boehringer Mannheim Biochemicals (Indianapolis, Indiana, USA). Tissue sections were counterstained with 2 $\mathrm{mg} / \mathrm{ml} \mathrm{4}$ 6-diamidine-2-phenylindole-dihydrocholride (DAPI) (Boehringer Mannheim Biochemicals) for $10 \mathrm{~min}$ at room temperature. Photomicrographs were recorded on $35-\mathrm{mm}$ film using a Nikon Optiphot microscope and UFX camera system (Nikon Inc., Melville, New York, USA) and transferred onto a Kodak Photo CD (Eastman Kodak). The images were digitally adjusted for contrast using Adobe Photoshop 3.0 (Adobe Systems Inc.).

Computer-aided image analysis. To quantify the extent of apoptosis in the rat lung, samples were studied by epifluorescence to view either TUNEL-positive nuclei $(590 \mathrm{~nm})$ or total DAPI-stained nuclei $(420 \mathrm{~nm})$. Images were captured with a CCD video camera. The captured images were analyzed using the Image 1 system (Universal Imaging, West Chester, Pennsylvania, USA), which is an IBM-based software applications package for quantitative morphometry and image analysis. All images were captured to a hard disk at black level and gain settings identical to those used with the CCD camera. Likewise, images were digitally thresholded using identical settings for each set of either DAPI- or TUNELfluorescent groups. The total number of cells (nuclei) or the number of TUNEL-positive cells in each field was determined in the object-counting mode. At least 25 fields were analyzed from at least two individual animals at each time point. The apoptotic index was calculated as the percent of TUNEL-positive apoptotic nuclei divided by the DAPI-staining nuclei.

Statistical analysis. Data are expressed as the mean \pm SEM. Differences in measured variables between experimental and control group were assessed using Student's $t$ tests. Statistical calculations were performed on a Macintosh personal computer using the Statview II Statistical Package (Abacus Concepts, Berkeley, California, USA). Statistical difference was accepted at $P<0.05$.

\section{Results}

Generation of recombinant adenovirus containing HO- 1 cDNA. A 1.0-kbp Xho I-HindIII fragment of the rat HO-1 cDNA clone $\mathrm{pRHO}-1$ (12), containing the entire coding region, 

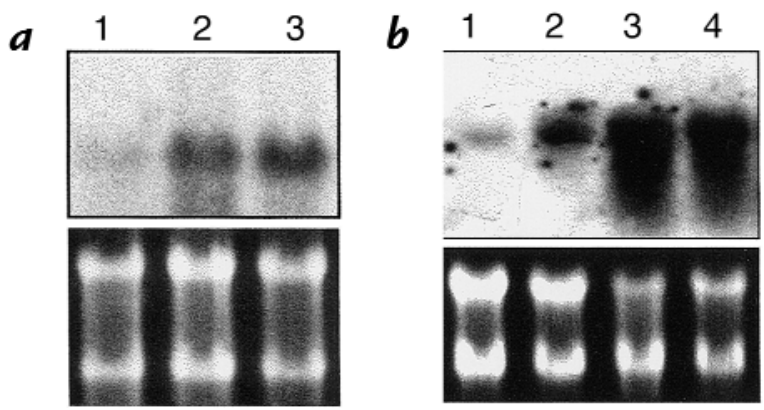

\section{Figure 4}

Effect of Ad5-HO-1 on hyperoxia-induced HO-1 mRNA expression. Total RNA was extracted from lung tissue after hyperoxia in the absence $(\boldsymbol{a})$ or presence $(\boldsymbol{b})$ of Ad5-HO-1 administration and analyzed for HO-1 mRNA expression by Northern blot analysis. (a) Lane 1: 0 h; lane 2: 48 h; lane 3 56 h. (b) Lane 1: AdV- $\beta$ Gal alone 72 h; lane 2: Ad5-HO-1 alone 72 h; lane 3: Ad5-HO-1 and hyperoxia $48 \mathrm{~h}$; lane 4: Ad5-HO-1 and hyperoxia $56 \mathrm{~h}$. Ethidium bromide staining of the same RNA is shown for loading control.

was cloned into plasmid pAC-CMVpLpA $(13,14)$. Recombinant adenoviruses containing the rat HO- 1 cDNA fragment, Ad5-HO-1, were generated by homologous recombination in 911 cells (15) cotransfected with the pAC-HO-1 recombinant plasmid and plasmid PJM17 (16).

Expression of HO-1 after Ad5-HO-1 administration. We first examined whether exogenous administration of HO- 1 by transgene delivery can induce HO-1 expression in the rat lung. Lungs were removed at 6 hours, 24 hours, 48 hours, 72 hours, 1 week, and 2 weeks after administration of Ad5-HO-1 (109 $\mathrm{pfu})$ and analyzed for HO-1 mRNA expression by Northern blot analyses. As shown in Figure 1 , we observed increased HO-1 mRNA levels as early as 6 hours, with peak levels at 48 hours to 72 hours of Ad5HO-1 administration. Increased HO-1 protein, as determined by Western blot analyses, was also observed in rats receiving Ad5-HO- 1 when compared with control rats receiving vehicle (Figure 2, lane 1) or AdV- $\beta \mathrm{G}$ al alone (Figure 2, lane 2). To further localize the expression of HO-1 protein, we performed immunohistochemical staining in lung sections after Ad5-HO-1 administration (Figure 3). Figure $3 a$ is a lung section from Ad5-HO-1-treated rats stained without primary antibody, but with secondary antibody alone as a control for nonspecific staining. As shown in Figure 3, $b$ and $d$, rats receiving vehicle alone exhibited negligible levels of HO-1 expression. However, marked staining of HO-1 protein was detected in the rat lung, especially bronchiolar epithelium, after Ad5-HO-1 administration (Figure $3 c$ ). Figure $3 e$ represents a higher magnification illustrating the high levels of $\mathrm{HO}-1$ protein expression in the bronchiolar epithelium after administration of Ad5-HO-1. Figure 4 illustrates the levels of HO1 mRNA expression in both control rats and rats receiving Ad5-HO-1. We observed increased HO-1 mRNA expression in control rats after hyperoxia, whereas rats receiving Ad5-HO-1 and exposed to hyperoxia exhibited accentuation of HO-1 mRNA expression compared with rats receiving Ad5-HO- 1 alone (Figure 4).

Effects of Ad5-HO-1 on byperoxia-induced lung injury. After successfully demonstrating the feasibility of increasing HO- 1 levels by exogenous administration of
Ad5-HO-1, we then sought to examine whether enhanced HO- 1 synthesis after gene transfer can confer protection against oxidant-induced lung injury. We first determined the amount of pleural effusion, a wellestablished and reproducible marker of hyperoxic lung injury, in rats receiving Ad5-HO-1. As shown in Figure 5 , rats receiving AdV- $\beta$ Gal or $10 \%$ glycerol (or vehicle control) alone exhibited marked increase in pleural effusion after 56 hours of hyperoxia exposure compared with negligible amounts in rats exposed to normoxia. However, rats receiving Ad5-HO-1 exhibited significantly less amounts of pleural effusion $(P<0.009)$ compared with rats receiving AdV- $\beta$ Gal or vehicle alone. We further examined whether rats receiving Ad5HO-1 exhibited attenuated lung injury by histological analyses. Figure $6 a$ demonstrates normal alveolar architecture of untreated control animals. Rats receiving Ad5-HO-1 alone also exhibited normal lung architecture with minimal inflammation at 56 hours of normoxia (Figure 6b). Marked lung hemorrhage, edema, alveolar septal thickening, influx of inflammatory cells, and fibrin deposition were observed in rats receiving vehicle alone after 56 hours of hyperoxia (Figure 6, $c$ and $e$ ); however, we observed significant preservation of lung architecture after 56 hours of hyperoxia in rats receiving Ad5-HO-1 (Figure 6, $d$ and $f$ ).

Effects of Ad5-HO-1 on byperoxia-induced lethality. Surgical sham animals and rats receiving either $10 \%$ glycerol or AdV- $\beta$ Gal all died by $66 \mathrm{~h}$ of hyperoxia exposure (Figure 7). We observed a significant level of tolerance against hyperoxia in rats receiving Ad5-HO-1, with approximately $66 \%$ of the animals surviving past 96 hours.

Effects of Ad5-HO-1 on antioxidant enzymes and ferritin. We examined whether overexpression of $\mathrm{HO}-1$ in rat lung by gene transfer of Ad5-HO-1 resulted in modulation of other antioxidant enzymes or ferritin. Figure 8 illustrates that the mRNAs for antioxidant enzymes Mn-SOD and

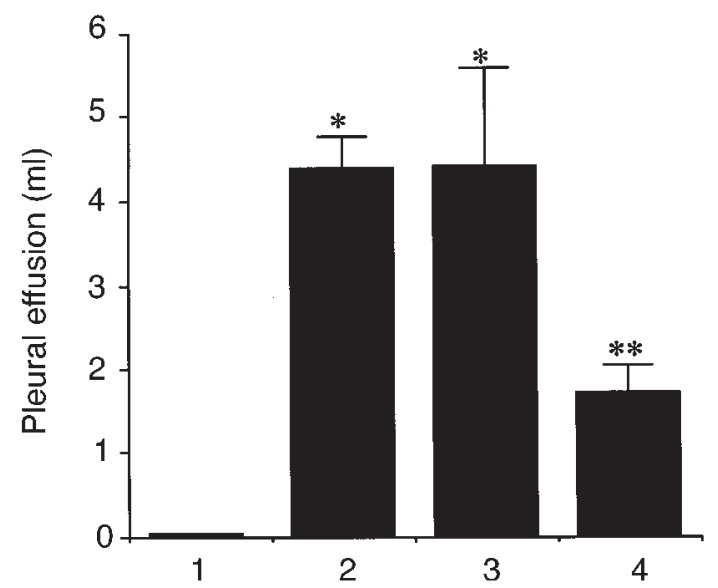

Figure 5

Pleural effusion volume after hyperoxia exposure. Pleural effusion volume was measured in rats exposed to $56 \mathrm{~h}$ of hyperoxia in the presence or absence of Ad5-HO-1. Data represent the mean value $\pm \mathrm{SE}$ of samples from $n$ rats. Bar 1: normoxia sham controls $(n=12)$; bar 2 : hyperoxia vehicle controls (glycerol; $n=16$ ); bar 3 : hyperoxia vector controls (AdV- $\beta$ Gal; $n=6)$; bar 4: hyperoxia Ad5-HO-1 $(n=14)$. ${ }^{*} P<0.0001$ compared with normoxia controls. ${ }^{*} P<0.009$ compared with vector or vehicle controls. 

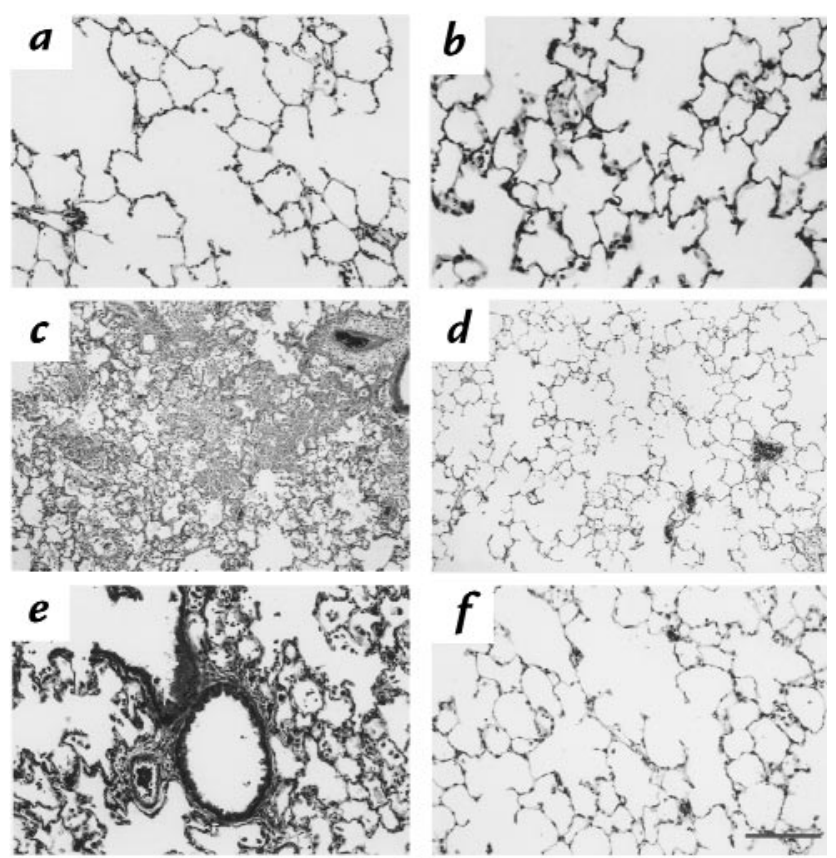

Figure 6

Histological analysis of rat lung after hyperoxia. Formalin-fixed sections of rat lungs were stained with hematoxylin and eosin. (a) Normoxia control. $\times 10$. (b) Ad5-HO-1 alone $56 \mathrm{~h}$. $\times 10$. (c) Vehicle control $56 \mathrm{~h} \mathrm{O}_{2}$ $\times 10$. (d) Ad5-HO-1 $56 \mathrm{~h} \mathrm{O}_{2} . \times 10$. (e) Vehicle control $56 \mathrm{~h} \mathrm{O}_{2} . \times 26$. (f) Ad5-HO-1 $56 \mathrm{~h} \mathrm{O}_{2} . \times 26$.

CuZn-SOD, and for L-ferritin and $H$-ferritin were not upregulated in rats receiving Ad5-HO-1 when compared with rats receiving $A d V-\beta G a l$ alone.

Ad5-HO-1 administration significantly attenuates byperoxiamediated neutrophil influx and lung apoptosis. To investigate the possible mechanisms of Ad5-HO-1-mediated protection against hyperoxia, we examined the inflammatory cell profile in the BAL of animals exposed to hyperoxia. It is well established that neutrophil influx in the BAL is of paramount importance in the development of hyperoxia-induced lung injury in in vivo models and in human patients with adult respiratory distress syndrome (ARDS) $(21,22)$. We hypothesized that Ad5-HO-1 may protect against lung injury by inhibiting neutrophil influx into the airways. Animals exposed to hyperoxia alone demonstrate a marked increase in neutrophil influx into the airways as assessed by BAL analysis (Figure 9). In marked contrast, rats exposed to hyperoxia after pretreatment of Ad5-HO-1 exhibited significant reductions in neutrophil influx when compared with rats receiving AdV- $\beta$ Gal or glycerol buffer alone. Another possible mechanism by which Ad5-HO-1 might exert its salutary effects would be by inhibiting apoptosis. Although the precise physiologic function of apoptosis in the lung has yet to be established, emerging data strongly suggest that the total lung apoptotic index can serve as a useful marker of lung injury in response to oxidative stress such as hyperoxia $(20,23)$. We have observed that rats exposed to hyperoxia alone exhibit a highly significant induction in the lung apoptotic index when compared with control rats in normoxia (Figure 10) $(P<0.05)$. In contrast, rats exposed to hyperoxia after pretreatment with Ad5-HO-1 demon- strate a significant reduction in the lung apoptotic index when compared with animals exposed to hyperoxia alone (Figure 10) $(P<0.001)$.

\section{Discussion}

A substantial body of evidence, accumulated in recent years, points to an important functional role for $\mathrm{HO}-1$ in providing cellular protection against oxidative stress. Both in vitro and in vivo data demonstrate that the induction of endogenous levels of $\mathrm{HO}-1$ in response to oxidant injury in turn protects the cells and tissues against subsequent oxidant-induced injury (8-11, 24, 25). The recent study by Poss and Tonegawa (26), demonstrating the increased susceptibility of HO1-null knockout mice to oxidative stress, further strengthens the emerging paradigm that $\mathrm{HO}-1$ is indeed an important molecule in the host's and cell's defense against oxidant stress. In particular, our laboratory has shown that endogenous expression of HO-1 is induced both in vivo and in vitro in response to hyperoxia exposure and that increasing levels of $\mathrm{HO}-1$ in cells can provide cytoprotection against hyperoxic injury in vitro $(6,11)$. We sought to examine whether HO-1 similarly can provide protection against hyperoxia in vivo.

We elected to use recombinant adenovirus to deliver exogenous HO-1 in a rat model of hyperoxia-induced lung injury. The use of adenovirus as a vector for lungdirected gene therapy has made significant progress in the area of lung biology, in particular in disease states such as cystic fibrosis. We show for the first time the feasibility of delivering exogenous HO-1 by gene transfer into the lung with successful expression of HO-1 mRNA and protein. Our data indicate that pretreatment with HO-1 transgene significantly attenuates hyperoxiainduced lung injury and increases survival in response to lethal hyperoxia. Although we cannot absolutely exclude a combined vector and $\mathrm{HO}-1$ effect, this is unlikely since we used vector and vehicle controls in our experiments. While $66 \%$ is a significant improvement in mortality, there is still $34 \%$ of animals that eventually succumb to the lethal concentration of oxygen exposure. It is not sur-

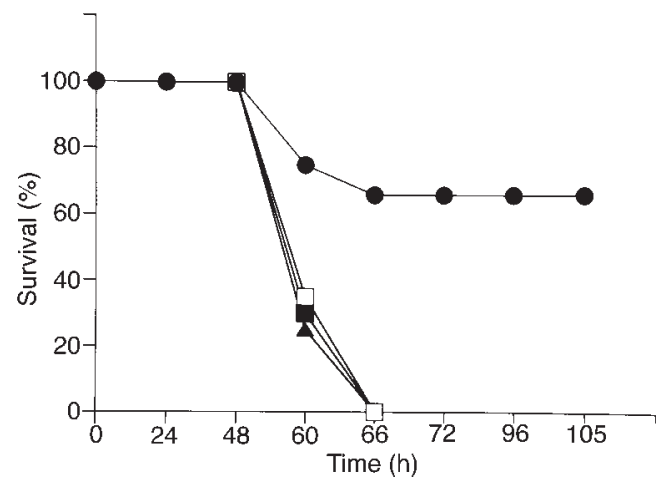

Figure 7

Survival curve of rats exposed to hyperoxia. Rats were exposed to $>99 \%$ $\mathrm{O}_{2}$ and monitored for survival. Vehicle-treated (glycerol; $n=12$ ), vectortreated (AdV- $\beta$ Gal; $n=6)$, and sham surgical rats $(n=4)$ died within $66 \mathrm{~h}$, whereas $66 \%$ of Ad5-HO-1-treated rats $(n=12)$ survived $>96$ h. Filled triangles: glycerol control; closed squares: AdV- $\beta \mathrm{Gal}$; open squares: sham surgery controls; closed circles: Ad5-HO-1. 


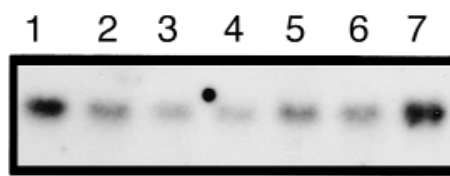

L-ferritin

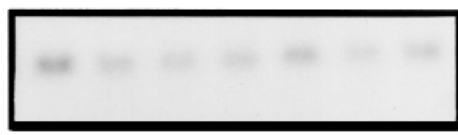

$H$-ferritin

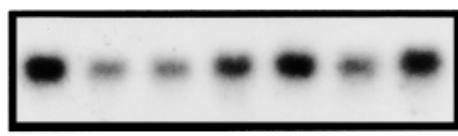

CuZn-SOD

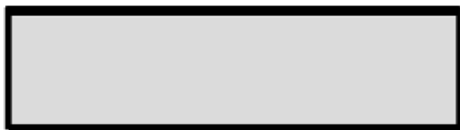

Mn-SOD

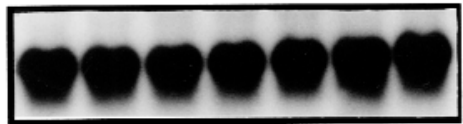

18s rRNA

\section{Figure 8}

Northern blot analyses of lung tissues after Ad5-HO-1 administration. Total RNA was extracted from lung tissue after Ad5-HO-1 administration and analyzed for the indicated mRNA expression by Northern blot analysis at the indicated times. Lane 1: vehicle control $72 \mathrm{~h}$; lane 2: $6 \mathrm{~h}$; lane 3: $24 \mathrm{~h}$; lane 4: $48 \mathrm{~h}$; lane 5: $72 \mathrm{~h}$; lane 6: 1 week; lane 7: 2 weeks. 18s rRNA hybridization is shown for normalization controls. $\mathrm{CuZn-SOD}$, copper-zinc superoxide dismutase; $M n-S O D$, manganese superoxide dismutase.

prising that in a diffuse lung injury model such as hyperoxia, which encompasses and involves complex and overlapping pathways, modulation of one specific molecule would have incomplete protection against a specific stimulus. This incomplete protection in terms of survival has also been observed in models of tolerance to hyperoxia involving stress response gene products such as heat shock proteins (27). Furthermore, in studies involving intratracheal administration of adenovirus, such as this model, there will be variability among animals in the delivered amount of virus, incorporation and expression of $\mathrm{HO}-1$, and differences in the immune response of rats.

In contrast to the conclusions reached herein, a recent study by Taylor et al. (28) has suggested that HO-1 does not provide protection against hyperoxic lung injury. In that study, intratracheal administration of hemoglobin into rats before hyperoxia exposure induced lung-specific expression of HO-1 and ameliorated subsequent lung injury due to oxygen toxicity. Inhibition of $\mathrm{HO}$ enzyme activity by tin-protoporphyrin, however, neither augmented hyperoxic lung injury nor reversed the protection conferred by hemoglobin. Based on these findings, they concluded that the protective effects of hemoglobin against hyperoxia were not due to HO-1, but perhaps were caused by direct induction of ferritin by hemoglobin. Certainly, reconciliation of the contradictory results within the two studies will require additional investigations. One potential explanation, however, is that hemoglobin has pleiotropic effects and activates multiple independent protective mechanisms. If these mechanisms are redundant, inhibition of an individual protective pathway may not be sufficient to reverse the ameliorative effects of hemoglobin. This idea is consistent with the results of Taylor et al. (28), who observed that hemoglobin stimulated $\mathrm{HO}-1$ and ferritin expression independent of $\mathrm{HO}$ activity. Ferritin, by virtue of its ability to sequester free iron in ferritin complexes, and thus remove a potent catalyst of hydroxyl radical formation, is proposed to protect against oxidative stress $(1,2)$. Administration of Ad5HO-1 provides for more direct assessment of the role of HO-1 independent of other antioxidant or protective functions (Figure 8). We observed that upregulating HO1 expression directly by Ad5-HO- 1 can provide protection against hyperoxia in vivo without affecting expression of antioxidant enzymes or ferritin. Furthermore, in contrast to the effects of Ad5-HO-1 on neutrophil influx (Figure 9), the ameliorative mechanism of hemoglobin apparently does not require antineutrophil or antioxidant activities. Finally, in our hands, and in contrast to the observations of Taylor et al. (28), administration of tinprotoporphyrin to animals consistently augments lung tissue injury in response to various oxidants $(9,11)$, including hyperoxia (29).

The precise mechanisms by which ectopic expression of HO-1 provided protection against lethal hyperoxia remains unclear. Our studies demonstrating that exogenous administration of HO-1-imparted anti-inflammatory and antiapoptotic effects are consistent with published reports of the effects of endogenous HO-1 overexpression. Willis et al. (30) recently reported that induction of endogenous HO-1 imparts potent antiinflammatory effects in vivo, and Soares et al. (31) reported that HO-1 serves as a potent antiapoptotic molecule in a model of inflammation in tissue transplantation. The by-products of $\mathrm{HO}$ catalysis, including $\mathrm{CO}$, ferritin from released iron, and bilirubin, represent attractive candidate molecules that may play key roles in mediat-

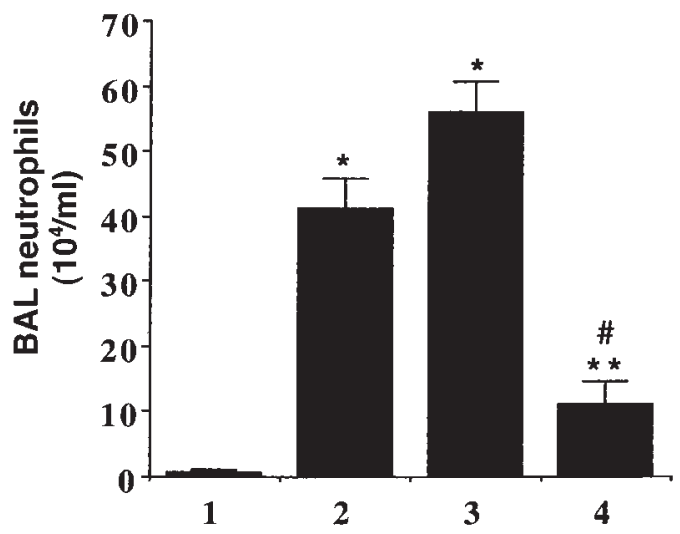

\section{Figure 9}

BAL cell count after hyperoxia. Differential cell counts for neutrophils were performed in the BAL fluid $56 \mathrm{~h}$ after hyperoxia in the presence or absence of Ad5-HO-1. Data represent the mean value \pm SE of lavage samples $(n=6)$. Bar 1 : normoxia sham controls $(n=10)$; bar 2 : hyperoxia vehicle controls (glycerol; $n=10$ ); bar 3 : hyperoxia vector controls (AdV$\beta \mathrm{Gal} ; n=6)$; bar 4: hyperoxia Ad5-HO-1 $(n=6)$. ${ }^{*} P<0.0001$ compared with normoxia controls. ${ }^{*} P<0.0006$ compared with vehicle controls. ${ }^{\#} P<0.0001$ compared with vector controls. BAL, bronchoalveolar lavage. 


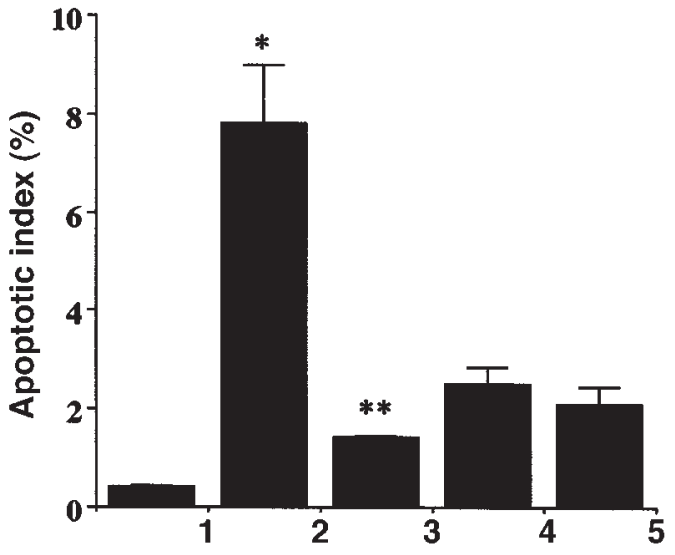

\section{Figure 10}

Effect of Ad5-HO-1 on hyperoxia-induced apoptosis in the rat lung. Lung sections from rats were analyzed for apoptotic signals by TUNEL-positive cells and costained with DAPI to determine the apoptotic index (number of TUNEL-positive cells per number of DAPI-stained cells) after $56 \mathrm{~h}$ of hyperoxia exposure. Bar 1: normoxia controls; bar 2: hyperoxia alone 56 h; bar 3: hyperoxia Ad5-HO-1 56 h; bar 4: Ad5-HO-1 alone 56 h; bar 5: AdV$\beta \mathrm{Gal}$ alone $56 \mathrm{~h}$. Data represent the mean value $\pm \mathrm{SE}$ of three rats. ${ }^{*} P<$ 0.05 compared with normoxia controls. ${ }^{*} P<0.001$ compared with hyperoxia alone. DAPI, 4'6-diamidine-2-phenylindole-dihydrocholride; TUNEL, terminal deoxynucleotide transferase-mediated dUTP nick end-labeling.

ing the protective effects of HO- 1 against oxidant-mediated lung injury. For example, Stocker et al. (32) have shown that bilirubin possesses potent antioxidant properties, which also have been demonstrated in vivo (33). The other formal possibility is the role of $\mathrm{CO}$, a gaseous molecule with biological activities similar to nitric oxide (34-36). Although the biological and physiological role of $\mathrm{CO}$ remains unclear in our model, the intriguing possibility that $\mathrm{CO}$, a nonreactive diffusible gas, could impart biological function warrants further studies in various models of oxidative stress, including hyperoxia. Our laboratory has recently observed that exogenous $\mathrm{CO}$ at low concentrations can provide tolerance against hyperoxic lung injury (29). Further studies are needed to examine the possible mechanisms by which $\mathrm{CO}$ mediates protection against hyperoxia.

In summary, we have demonstrated the feasibility of delivering exogenous HO-1 by an adenovirus gene therapy approach, with successful incorporation and expression of the gene product. We report here that the induction of $\mathrm{HO}-1$ by exogenous delivery provided physiologic protection against lethal hyperoxia. We have also explored in this study possible mechanisms to explain the protective functions attributed to HO- 1 in hyperoxia-induced lung injury. This study raises the intriguing possibility of the potential therapeutic use of $\mathrm{HO}-1$, not only in lung disorders such as ARDS and sepsis, but also in other inflammatory disease states.

\section{Acknowledgments}

The work by A.M.K. Choi was supported by National Institutes of Health (NIH) grants RO1 HL-55330, RO1 HL-60234, and R01 AI-42365, and an American Heart Association Established Investigator Award. J. Alam was supported by NIH grant DK-
43135. J.K. Kolls was supported by grant R29AA10384 from the National Institute on Alcohol Abuse and Alcoholism and by the Cystic Fibrosis Foundation. L.L. Mantell was supported in part by grants from the American Lung Association and the Stony Wold-Herbert Fund.

1. Choi, A.M.K., and Alam, J. 1996. Heme oxygenase-1: function, regulation and implication of a novel stress-inducible protein in oxidant-induced lung injury. Am. J. Respir. Cell Mol. Biol. 15:9-19.

2. Maines, M.D. 1997. The heme oxygenase system: a regulator of second messenger gases. Annu. Rev. Pharmacol. Toxicol. 37:517-554.

3. McCoubrey, W.K., Huang, T.J., and Maines, M.D. 1997. Isolation and characterization of a cDNA from the rat brain that encodes hemoprotein heme oxygenase-3. Eur. J. Biochem. 247:725-732.

4. Tenhunen, R., Marver, H.S., and Schmidt, R. 1970. The enzymatic catabolism of hemoglobin: stimulation of microsomal heme oxygenase by hemin. J. Lab. Clin. Med. 75: 410-421.

5. Keyse, S.M., and Tyrrell, R.M. 1989. Heme oxygenase is the major 32-kDa stress protein induced in human skin fibroblasts by UVA radiation, hydrogen peroxide, and sodium arsenite. Proc. Natl. Acad. Sci. USA. 86:99-103.

6. Lee, P.L., et al. 1996. Regulation of heme oxygenase-1 expression in vivo and in vitro in hyperoxic lung injury. Am. J. Respir. Cell Mol. Biol. 14:556-568.

7. Applegate, L.A., Luscher, P., and Tyrell, R.M. 1991. Induction of heme oxygenase: a general response to oxidant stress in cultured mammalian cells. Cancer Res. 51:974-978.

8. Nath, D.A., et al. 1992. Induction of heme oxygenase is a rapid, protective response in rhabdomyolysis in the rat. J. Clin. Invest. 90:267-270.

9. Otterbein, L.E, Sylvester, S.L., and Choi, A.M.K. 1995. Hemoglobin provides protection against lethal endotoxemia in rats: The role of heme oxygenase-1. Am. J. Respir. Cell Mol. Biol. 13:595-601.

10. Abraham, N.G., et al. 1995. Transfecton of the human heme oxygenase gene into rabbit coronary microvessel endothelial cells: protective effects against heme and hemoglobin toxicity. Proc. Natl. Acad. Sci. USA. 92:6798-6802.

11. Lee, P.J., Alam, J., Wiegand, G.W., and Choi, A.M.K. 1996. Overexpression of heme oygenase- 1 in human pulmonary epithelial cells results in cell growth arrest and increased resistance to hyperoxia. Proc. Natl. Acad. Sci. USA. 93:10393-10398.

12. Shibahara, S., Muller, R., Taguchi, H., and Yoshida, T. 1985. Cloning and expression of cDNA for rat heme oxygenase. Proc. Natl. Acad. Sci. USA . 82:7865-7869.

13. Graham, F.L., and Prevec, L. 1991. Manipulation of adenovial vectors in molecular biology. Gene transfer and expression protocols. 7:109-128.

14. Gomez-Foix, A.M., et al. 1992. Adenovirus-mediated transfer of the muscle glycogen phosphorylase gene into hepatocytes confers altered regulation of glycogen metabolism. J. Biol. Chem. 267:25129-25134.

15. Fallaux, F.J., et al. 1996. Characterization of 911: a new helper cell line for the titration and propagation of early region 1-deleted adenoviral vectors. Hum. Gene Ther. 7:215-222.

16. McGrory, W.J., Bautista, D.S., and Graham, F.L. 1988. A simple technique for the rescue of early region I mutations into infectious human adenovirus type 5. Virology. 163:614-617.

17. Kolls, J., Peppel, K., Silva, M., and Beutler, B. 1994. Prolonged and effective blockade of tumor necrosis factor activity through adenovirus-mediated gene transfer. Proc. Natl. Acad. Sci. USA. 91:215-219.

18. Camhi, S., Alam, J., Otterbein, L.E., Sylvester, S.L., and Choi, A.M.K. 1995. Induction of heme oxygenase- 1 gene expression by lipopolysaccharide is mediated by AP-1 activation. Am. J. Respir. Cell Mol. Biol. 13:387-398.

19. Otterbein, L., et al. 1997. Mechanism of hemoglobin-induced protection against endotoxemia in rats: a ferritin-independent pathway. Am.J. Physiol. 272:L268-L275.

20. Mantell, L.L., et al. 1997. Unscheduled apoptosis during acute inflammatory lung injury. Cell Death Differ. 4:600-607.

21. Steinberg, K.P., et al. 1994. Evolution of bronchoalveolar cell populations in the adult respiratory distress syndrome. Am. J. Respir. Crit. Care Med. 150:113-122.

22. Choi, A.M.K., Sylvester, S.L., Otterbein, L.E., and Holbrook, N.J. 1995. Molecular responses to hyperoxia in vivo: Relationship to increased tolerance in aged rats. Am. J. Respir. Cell Mol. Biol. 13:74-82.

23. Otterbein, L.E., et al. 1998. Pulmonary apoptosis in aged and oxygen-tolerant rats exposed to hyperoxia. Am. J. Physiol. 275:L14-L20.

24.Vile, G.F., Basu-Modak, S., Waltner, C., and Tyrrell, R.M. 1994. Heme oxygenase 1 mediates an adaptive response to oxidative stress in human skin fibroblasts. Proc. Natl. Acad. Sci. USA. 91:2607-2610.

25. Vile, G.F., and Tyrrell, R.M. 1994. Oxidative stress resulting from ultraviolet $\mathrm{A}$ irradiation of human skin fibroblasts leads to a heme oxygenasedependent increase in ferritin. J. Biol. Chem. 268:14678-14681.

26. Poss, K.D., and Tonegawa, S. 1997. Reduced stress defense in heme oxygenase 1 deficient cells. Proc. Natl. Acad. Sci. USA. 94:10925-10930.

27. Wong, H.R., Menendez, I.Y., Ryan, M.A., Denenberg, A.G., and Wisp, J.R. 1998. Increased expression of heat shock protein-70 protects A549 cells 
against hyperoxia. Am.J. Physiol. 275:L836-L841.

28. Taylor, J.L., Carrawy, M.S., and Piantadosi, C.A. 1998. Lung-specific induction of heme oxygenase- 1 and hyperoxic lung injury. Am. J. Physiol. 18:L582-L591.

29. Otterbein, L.E., Mantell, L.L., and Choi, A.M. 1999. Carbon monoxide provides protection against hyperoxic lung injury. Am. J. Physiol. 276: L1-L7.

30. Willis, D., Moore, A.R., Frederick, R., and Willoughby, D.A. 1996. Heme oxygenase-1. A novel target for the modulation of the inflammatory response. Nat. Med. 2:87-90.

31. Soares, M. P., et al. 1998. Expression of heme oxygenase-1 (HO-1) can determine cardiac xenograft survival. Nat. Med. 4:1073-1077.

32. Stocker, R., Yamamoto, Y., McDonagh, A.F., Glazer, A.N., and Ames, B.N
1987. Bilirubin is an antioxidant of possible physiological importance. Science. 235:1043-1046.

33. Llesuy, S., and Tomaro, M. 1994. Heme oxygenase and oxidative stress. Evidence of involvement of bilirubin as physiological protector against oxidative damage. Biochim. Biophys. Acta. 1223:9-14.

34. Kharitonov, V.G., Sharma, V.S., Pilz, R.B., Magde, D., and Koesling, D. 1995. Basis of guanylate cyclase activation by carbon monoxide. Proc. Natl. Acad. Sci. USA. 92:2568-2571.

35. Marilena, G. 1997. New physiological importance of two classic residual products: carbon monoxide and bilirubin. Biochem. Mol. Med. 61:136-142.

36. Verma, A., Hirsch, D.J., Glatt, C.E., Ronnett, G.V., and Synder, S.H. 1993. Carbon monoxide: a putative neural messenger. Science. 259:381-384. 